Recherches en histoire de l'art, histoire des civilisations, archéologie, anthropologie et muséologie

$15 \mid 2020$

Cahier $\mathrm{n}^{\circ} 15$

\title{
De la violence au cadavre : étude d'une mort genrée
}

From violence to corpse, study of a gendered death

\section{Anaelle Lahaeye}

\section{OpenEdition}

Journals

Édition électronique

URL : http://journals.openedition.org/cel/8242

DOI : $10.4000 /$ cel.8242

ISSN : 2262-208X

Éditeur

École du Louvre

\section{Référence électronique}

Anaelle Lahaeye, «De la violence au cadavre : étude d'une mort genrée », Les Cahiers de l'École du Louvre [En ligne], 15 | 2020, mis en ligne le 31 octobre 2020, consulté le 05 novembre 2020. URL : http://journals.openedition.org/cel/8242 ; DOI : https://doi.org/10.4000/cel.8242

Ce document a été généré automatiquement le 5 novembre 2020.

\section{(c) $($ i) $(9)$}

Les Cahiers de l'École du Louvre sont mis à disposition selon les termes de la licence Creative Commons Attribution - Pas d'Utilisation Commerciale - Pas de Modification 4.0 International. 


\section{De la violence au cadavre : étude d'une mort genrée}

From violence to corpse, study of a gendered death

Anaelle Lahaeye

1 La façon de traiter, de représenter et de considérer les dépouilles est signifiante. Elle est intimement liée au rapport des sociétés à la mort, mais aussi au corps, et par son entremise, au genre. Dans cette logique s'inscrit l'étonnant succès du motif de la belle morte dans la France du XIX siècle. Il abonde au sein des arts visuels et de la littérature, au point de créer un imaginaire qui essaime aussi bien dans les journaux de mode que sur les cimaises du Salon. Une vogue d'autant plus surprenante qu'elle s'inscrit à contre-courant de l'évolution globale du reste des œuvres présentant des défunts. Un réalisme croissant est en effet perceptible dans le traitement de ces derniers au fil des décennies.

2 Ce paradoxe s'explique notamment par le caractère genré des normes de représentation des cadavres. Une dimension qui rend le motif propice à l'expression de stéréotypes liés peu ou prou à la violence. Les belles mortes sont donc un exemple de choix pour questionner la façon dont l'art est à la fois un espace de matérialisation et de dépassement des systèmes de pensée d'une époque.

\section{Une beauté surprenante}

«Au croisement des émotions et des représentations, de l'imaginaire et des sensibilités ${ }^{1}$ ", l'appréhension de la mort par les sociétés humaines ne peut pas être pensée de façon uniforme. Au fil des époques, cette appréhension a varié, en témoigne la grande diversité des représentations artistiques de la mort. Au sein de ces dernières, le motif du cadavre a une place à part. Nombreuses sont les thématiques et les genres picturaux qui le sollicitent : scènes de bataille, peintures religieuses, veillées funèbres, etc. Cette omniprésence en fait un motif banal et pourtant loin d'être anodin par l'imaginaire 
macabre qu'il convoque. L'évolution des normes de représentation des défunts participe de cette oscillation.

4 Différentes caractéristiques sont utilisées par les artistes pour spécifier le statut d'un corps défunt: la carnation, la position, l'expression, auxquelles on peut adjoindre l'interaction avec les autres protagonistes, les blessures sanglantes et la décomposition des chairs. Or chacun de ces traits peut se matérialiser dans un vaste spectre de représentations ${ }^{2}$. La simple carnation, par exemple, va d'une peau un peu pâle à une teinte cireuse qui ne laisse plus de doute sur le trépas. Ainsi, pour schématiser, nous pourrions diviser les différentes dépouilles que l'on retrouve en peinture en trois catégories: les cadavres, les corps morts et les belles morts. Ce que nous appelons cadavre est une représentation qui ne cache rien des différentes manifestations de la thanatomorphose $\mathrm{s}^{3}$. Au contraire, un corps mort euphémise la réalité de cette dernière pour la rendre moins dérangeante. Exit donc les marques de la décomposition, au profit de conventions moins provoquantes, comme le sommeil ou l'affliction des autres protagonistes de l'œuvre. La belle mort va plus loin en utilisant les quelques caractéristiques de la mort qui magnifient le corps, tout en oblitérant ses autres manifestations. Suivant les époques et les espaces culturels, les normes de représentations des défunts vont donc varier du tout au tout.

En France, dès la fin du XVII ${ }^{e}$ siècle, les codes de bienséance s'imposent, faisant du cadavre un "véritable défi représentatif ${ }^{4}$ ». Charles Alphonse Du Fresnoy invite ainsi les artistes à «fuir les choses barbares, rudes à la vue [...] comme aussi les choses impudiques, sordides, malséantes ${ }^{5} »$. Les œuvres antérieures sont relues à l'aune de ce jugement et les licences macabres des artistes sont remises en cause ${ }^{6}$. Au siècle suivant, le refus croissant de la violence dans les arts et le développement de la théorie du beau idéal s'opposent encore davantage à la représentation de dépouilles réalistes. Bien que la mimesis demeure une des bases des théories esthétiques de cette époque, c'est vers l'idéalité que le néoclassicisme l'oriente. Un certain nombre de résistances émergent cependant durant le XIX ${ }^{\mathrm{e}}$ siècle. Le traitement des défunts tend en effet à être de plus en plus proche de la thanatomorphose. Ce cheminement accuse néanmoins des disparités en fonction du statut des œuvres, de la thématique abordée et du genre de dépouilles.

6 Les dépouilles féminines sont d'une part peu nombreuses ${ }^{7}$ et d'autre part elles entrent en grande majorité dans la catégorie des belles morts. Un socle de caractéristiques communes les uni : la jeunesse, la beauté, une pose alanguie, mais surtout aucune marque de cadavérisation, si ce n'est le teint livide (très prisé au XIX siècle) et l'expression apaisée du sommeil. Le titre et le sujet de l'œuvre sont donc les seuls indices à disposition du spectateur pour comprendre le véritable état de la femme représentée. La Jeune martyre de Paul Delaroche $(1855)^{8}$ est un exemple de ce modèle (fig. 1). La défunte flotte à la surface des flots, créant une vision onirique. La pâleur de sa peau est accentuée par la lumière émanant de son auréole et sa chevelure comme sa robe ondoient doucement au fil de l'eau. Ce type d'œuvre a beaucoup de succès à la période qui nous intéresse, comme en témoigne Atala au tombeau $(1808)^{9} \mathrm{~d}$ 'Anne-Louis Girodet. À sa suite, de nombreux artistes vont reprendre le motif, certains allant même jusqu'à se spécialiser dans sa représentation. James Bertrand est ainsi décrit par Maurice du Seigneur comme le " peintre ordinaire des héroïnes à l'agonie, suicidées ou trépassées ${ }^{10} »$. Sa peinture Chrétiens retirant du Tibre les corps des martyrs (1845) ${ }^{11}$ 
présente ainsi une superbe jeune femme, totalement exempte de traces de la mort par noyade à laquelle elle vient pourtant de succomber.

Fig. 1.

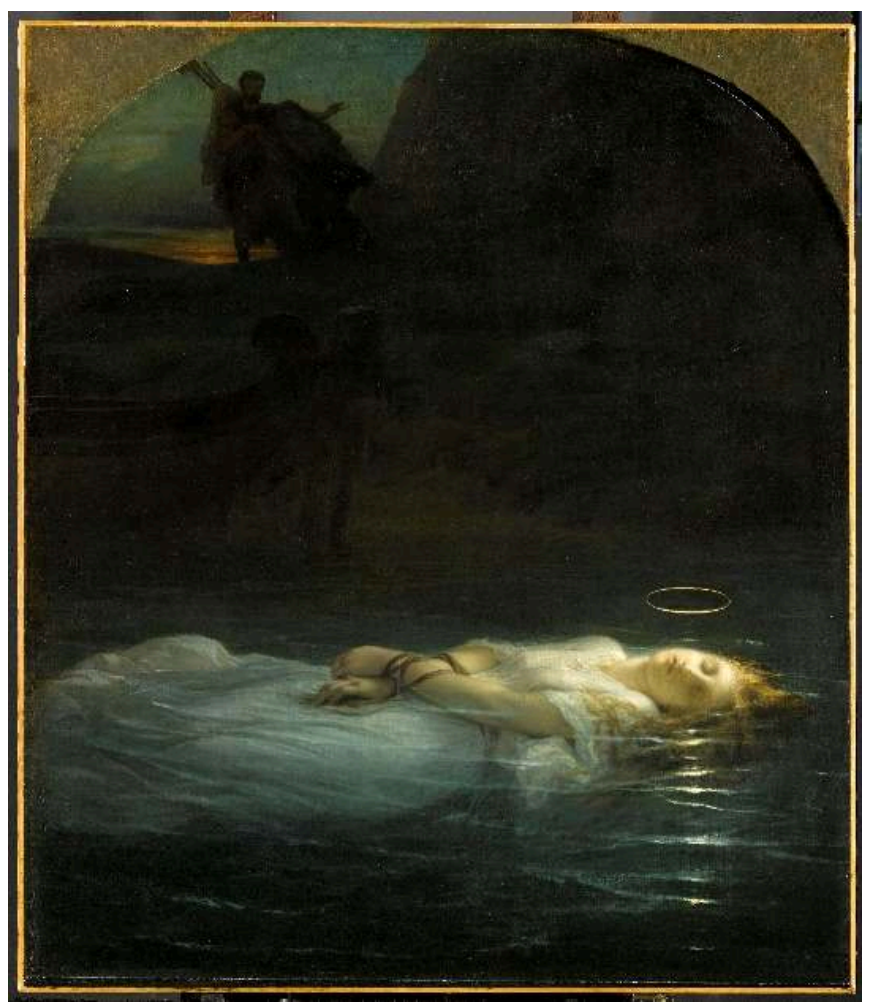

Paul Delaroche, La Jeune martyre, 1855, huile sur toile, Paris.

๑) RMN-Grand Palais (musée du Louvre)/René-Gabriel Ojéda

7 La prédominance de ce type d'œuvres est éloquente de l'enfermement des défuntes dans le registre de la belle mort. Il existe bien sûr quelques cas de cadavres féminins dans les peintures de l'époque. On peut citer Têtes de suppliciés (1818) de Théodore Géricault $^{12}$, ou encore Le Meurtre (1867) de Paul Cézanne ${ }^{13}$, pour les plus connues. Mais ce sont des exceptions comparé à la masse des belles mortes; en outre ces œuvres ne furent pas exposées. La spécificité des belles morts féminines est d'autant plus étonnante qu'elle dénote une résistance vis-à-vis de l'évolution du motif vers le cadavre, de plus en plus réaliste, à cette époque. Les belles mortes, ainsi qu'on peut les appeler, demeurent soumises à l'injonction de beauté prônée par Lessing et le néoclassicisme en général: "Tout autre objet possible des arts plastiques, s'il est inconciliable avec la beauté, doit être complètement écarté et, s'il peut se concilier avec elle, doit du moins lui être subordonné ${ }^{14}$ ». Ce biais de représentation devient presque aberrant pour les œuvres dont le sujet ne s'attache pas à une belle femme ou à une femme dont le trépas fait suite à un meurtre.

8 Tout comme les caractéristiques qui signalent la thanatomorphose, les marques de violences physiques sont complétement oblitérées de la plupart de ces œuvres. Un exemple très parlant est offert par la toile Néron devant le cadavre d'Agrippine (1824) du peintre Eugène Appert $t^{15}$ (fig. 2). Elle prend pour thème le matricide de Néron, relaté par Tacite. L'empereur, après avoir ordonné à ses soldats d'assassiner Agrippine, vient observer la dépouille de sa mère. Cette dernière, très belle, ne présente aucune 
blessure, alors même qu'elle est à demi nue et censée avoir succombé à plusieurs coups de couteaux. Une oblitération de la violence physique quasiment systématique lorsqu'il s'agit d'œuvres présentant des belles mortes. Pourtant les causes de décès de ces dernières sont généralement liées à un crime. Les belles mortes sont pour beaucoup de belles victimes, qu'il s'agisse de martyres religieux, de romances tragiques, ou d'évènements historiques funèbres. La bienséance pourrait expliquer cette absence de marques de sévices. Néanmoins, dès le début du siècle puis tout du long, les artistes représentent des cadavres masculins particulièrement meurtris, à l'instar des œuvres Napoléon sur le champ de bataille d'Eylau (1808) ${ }^{16} \mathrm{~d}^{\prime}$ Antoine Jean Gros ou celle d'Henri Regnault Exécution sans jugement sous les rois Maures de Grenade (1870) ${ }^{17}$.

Fig. 2.

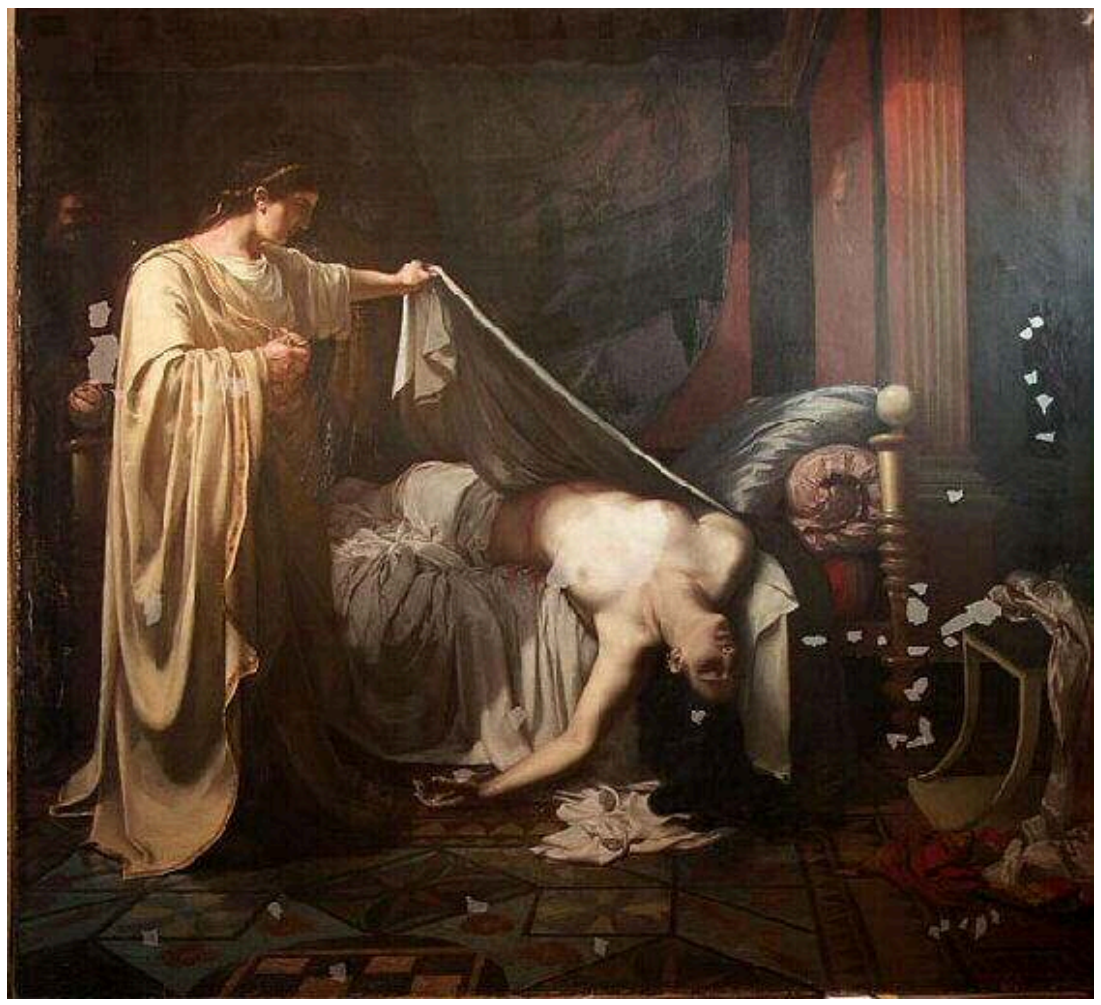

Eugène Appert, Néron devant le cadavre d'Agrippine, 1824, huile sur toile, Montauban.

(c) Montauban, musée Ingres (C) Direction des musées de France, 2004 (c) Roumagnac Guy

À l'inverse, les quelques dépouilles masculines qui entrent dans le champ de la belle mort reprennent les codes associés aux défuntes ${ }^{18}$. Des œuvres comme Apollon et Cyparisse de Jean-Pierre Granger $(1816)^{19}$ ou La Mort d'Abel de Jules-Élie Delaunay $(1856)^{20}$ présentent des corps pâles et fluets dans des poses alanguies qui s'apparentent d'avantage à la figure de l'androgyne qu'aux musculatures imposantes des guerriers néoclassiques. Dans ces deux œuvres, Abel et Cyparisse sont respectivement victimes de la violence de Caïn et d'Apollon. Pourtant aucune marque de sévices n'est visible (hormis de légères traces de sang sous le cadavre d'Abel). Deux exemples qui confirment les normes implicites qui entourent la représentation des défuntes, à savoir leur soumission à l'injonction de beauté dont découle l'effacement de toute visibilité des sévices. Mort et violence sont ainsi bien dissimulées sous le vernis de la beauté, au profit d'une esthétique qui va se répandre au-delà du champ artistique. 


\section{Des hagiographies aux gravures de mode}

10 Nous nous contenterons donc d'évoquer ses ramifications dans la religion chrétienne, qui émergent au sein des hagiographies du XIV ${ }^{\mathrm{e}}$ siècle. Les textes relatant le trépas des saints présentent en effet la beauté des dépouilles et leur non-corruption ${ }^{21}$ comme une preuve de grâce divine. Après les difficultés de la vie terrestre et les affres de l'agonie, leurs cadavres rayonnent et exhalent une odeur agréable, signe de leur accession au paradis céleste. Initialement réservé aux saints, ce type de récit perd progressivement son caractère exceptionnel et suit le regain de foi qui s'opère au début du XIX ${ }^{e}$ siècle. La beauté physique se veut alors l'écho de la perfection morale et de la foi du défunt. L'historienne Anne $\mathrm{Carol}^{22}$ a émis l'hypothèse d'une forme de re-sacralisation de la mort après la remise en cause de la religion lors de la Révolution française.

11

Ces origines entrent en écho avec les profondes modifications qui s'opèrent dans l'appréhension de la mort au sein la société. Comme l'ont montré les historiens des mentalités ${ }^{23}$ puis des représentations ${ }^{24}$, le XIXe siècle est une période charnière. D'une part, les aspects les plus terribles du trépas tendent de plus en plus à se dissimuler à la vue des vivants ${ }^{25}$ (éloignement des cimetières, règlementations strictes des salles de dissection, des abattoirs et des exécutions, etc.). La morgue, ouverte de 1804 à 1907, est un des derniers lieux qui permet de voir des dépouilles. D'autre part, le culte ostentatoire des défunts dans le cercle intime connait un succès sans précédent. L'engouement pour l'embaumement, les derniers portraits et les visites au cimetière en sont les traces ${ }^{26}$. Mais cette ostentation de la mort est moins celle du cadavre que d'une dépouille esthétisée, lavée, maquillée et débarrassée de toutes traces de thanatomorphose. Un double mouvement de répulsion et de sacralisation du corps mort est ainsi perceptible, le second s'accompagnant d'une intense fascination pour les représentations de belles morts.

Beaucoup de journaux privés évoquent ainsi cette beauté des défunts: "Nous sommes au temps des belles morts, celle de Madame de Villeneuve a été sublime ${ }^{27}$ ", «Avez-vous regardé sourire le cadavre? Tout à l'heure il râlait, se tordait, étouffait ; Maintenant il rayonne $e^{28}$ ", ou encore " elle paraissait bien plus grande et bien plus belle que vivante, avec ce long voile blanc qui lui descendait jusqu'aux pieds ${ }^{29} »$. Cet attrait se retrouve également dans de nombreux romans. Léo Lespès décrit dans Les Yeux verts de la Morgue (1846) le « corps d'une jeune fille d'une rare beauté. La pauvre enfant avait l'air d'être endormie. La blancheur de sa peau tranchait sur le noir de la pierre sur laquelle on avait jeté son corps, et donnait à son immobilité une grâce sans égal ${ }^{30}$ ». Théophile Gautier dans La Morte amoureuse (1836) compare quant à lui une défunte à « une statue d'albâtre faite par quelque sculpteur habile pour mettre sur un tombeau de reine, ou encore une jeune fille endormie sur qui il aurait neigé ${ }^{31} »$.

De cette omniprésence émerge une norme de beauté générale. Comme le souligne Amandine Malivin «le morbide devient alors une véritable valeur et une variété du beau $^{32}$ ». La blancheur du teint et les silhouettes fluettes sont mises à l'honneur, tandis qu'avoir une apparence moribonde devient source de compliments : «cette princesse Belgiojoso avait le visage d'un ovale remarquable par la pureté de ses lignes mais d'une pâleur étrange, qui lui donnait, le soir surtout, l'apparence d'une belle morte qui marchait ${ }^{33}$ ». Cette inclinaison surprenante des normes de beauté féminine a 
notamment été étudiée par Carolyn Day ${ }^{34}$ et Bram Dijkstra ${ }^{35}$. Elles ont montré l'attrait progressif pour une beauté malade, voir une beauté cadavérique, qui traverse le siècle et se retrouve dans les gravures de mode. Cette esthétique est d'ailleurs encouragée par la diffusion des derniers portraits puis des photographies post-mortem ${ }^{36}$, et reprise par les célébrités en vogue. Citons les étonnantes photographies de Sarah Bernhardt dans le cercueil qu'elle gardait dans sa loge et qui la suivait dans toutes ses tournées ${ }^{37}$. Un exemple quelque peu extrême, mais représentatif de l'impact et de l'importance du stéréotype de la belle morte au XIX ${ }^{\mathrm{e}}$ siècle.

14 Les belles mortes n'ont cependant pas qu'une influence esthétique, le motif étant porteur de nombreuses symboliques. En effet, il associe deux idées opposées, la beauté et la mort (qui est par essence la décomposition des formes et de l'unité esthétique). Ces corps incarnent de fait un moment d'hésitation, durant lequel la beauté est sublimée par sa proche corruption. Ils ne portent de la mort que les traces les plus agréables et échappent pour un temps à la nature. Une beauté figée qui porte une charge érotique très forte, appuyée par des choix de représentations suggestifs de la part des artistes. Nombreuses sont les belles mortes en partie dénudées ou vêtues simplement de tuniques transparentes, dans des poses souvent lascives. Si beaucoup de peintures de cette période présentent également des femmes dévêtues, le fantasme qu'implique la belle morte est légèrement plus insidieux. Ces dernières sont à la merci $\mathrm{du}$ spectateur, ce qui engendre une violence symbolique. Rappelons qu'une des raisons du scandale du Déjeuner sur l'herbe (1863) d'Édouard Manet ${ }^{38}$ est le regard de Victorine Meurent. Cette dernière, en fixant le spectateur, empêche le voyeurisme habituel des nudités picturales. Au contraire, la défunte est offerte : nul besoin de lui faire la cour, nul besoin de consentement et nulle résistance. Morte, elle ne parle pas, n'a pas d'identité ou de caractère. Elle incarne la beauté féminine soumise aux regards et aux fantasmes.

Ces représentations sont également un moyen efficace de dédramatiser la mort en masquant la réalité de la décomposition. Elles permettent ainsi une approche plus douce de quelque chose d'inconcevable, l'anéantissement de l'être. Cette sublimation des motifs jugés hideux ou dégoutants a d'ailleurs longtemps été le symbole d'une excellence artistique. Néanmoins, la confusion est dangereuse. Ces femmes sont-elles seulement des cadavres ? Il n'y a quasiment aucune différence entre les représentations des belles mortes et celles des belles endormies. Les premières ne portant pas ou peu d'indices du trépas, les deux motifs sont totalement interchangeables. Dans l'œuvre d'Henri Gervex Avant l'opération (1887) ${ }^{39}$, on retrouve clairement le modèle de la belle morte. La malade, particulièrement pâle, semble endormie, allongée nue et couverte à demi d'un drap. L'ambivalence avec les belles mortes est d'ailleurs accentuée par l'imaginaire visuel des scènes de dissection dont l'œuvre est inspirée. Cette confusion crée un sentiment d'irréalité autour du trépas de ces jeunes femmes, dont l'absence de marques de sévices annihile la violence dont elles ont été l'objet.

Elisabeth Bronfen ${ }^{40}$ et Lisa Downing ${ }^{41}$ ont également souligné l'importance symbolique de la position de ces dépouilles. Dans beaucoup d'œuvres, la victime est allongée au sol ou en train de chuter. Le meurtrier, presque systématiquement masculin, est quant à lui debout en train de l'observer. Une figure d'observateur ou de voyeur qui encourage les spectateurs et par lequel un rapport de supériorité s'instaure, accru par l'écart entre le vivant et le mort. Qu'un artiste choisisse de représenter un cadavre et non l'acte de violence en lui-même n'est en effet pas anodin. En confrontant le spectateur à la mort, 
le peintre accentue le caractère dramatique de la violence et instaure un rapport de domination entre le meurtrier et la victime, mais aussi entre le spectateur et le cadavre. L'iconographie des meurtrières comme Judith ou Charlotte Corday offre un contrepied intéressant. Dans cette dernière, ce sont les femmes qui prennent un rôle dominant tandis que les hommes prennent la place des victimes. Or leurs dépouilles sont rarement érotisées, et portent plus volontiers les marques des sévices. Une différence qui met au jour la dimension genrée de traitement des cadavres soumis à la violence.

\section{Dévoiements et dépassements du motif}

Bien que majoritaires, les belles mortes ne sont pas la seule voie de représentation des défuntes à notre période, loin s'en faut. Le XIX ${ }^{e}$ siècle est parcouru d'une puissante recherche de réalisme. L'engouement pour la peinture sur le motif, le souci de la vérité historique et l'importance donnée au respect de l'anatomie en témoignent. Afin de parfaire l'étude des figures, des cours de dissection sont même instaurés à l'école des Beaux-Arts ${ }^{42}$. Cet attrait entre en contradiction avec les normes du beau idéal prônées par le néoclassicisme au début du siècle. Si ces dernières ont permis aux belles mortes d'éclore, de nouvelles voix s'élèvent pour bannir l'idéalisation des corps.

Comme l'attestent les Têtes de suppliciés (1818) de Théodore Géricault, cette recherche de réalisme s'applique aux cadavres féminins. Une recherche qui va pousser l'artiste à se rendre à la morgue de l'hôpital de La Salpêtrière, en plus d'une étude sur modèle vivant. Deux têtes coupées se partagent l'espace de la toile, celles d'un homme et d'une femme. Une même violence préside la représentation des deux figures, des plaies sanguinolentes aux subtiles nuances de carnation allant du beige au vert. Si la défunte présente une expression endormie, la restriction à sa seule tête empêche tout érotisme de s'immiscer au sein de la composition. De fait, la toile ne cache rien de la trivialité et de la violence du trépas de la jeune femme. Cette tonalité macabre se retrouve dans Le Meurtre (1867) de Paul Cézanne. Le spectateur y est confronté directement à l'horreur de l'acte et au faciès de la victime, déformé par la douleur, la bouche ouverte figée dans un cri muet. Ici encore, nulle place pour la beauté. Des exemples percutants qui n'auront cependant pas d'impact significatif sur la scène artistique. Peu ou pas exposées, ces œuvres étaient sans doute trop loin des standards pour pouvoir exister autrement que dans des cercles privés.

Pour autant, les toiles présentées au Salon connaissent aussi les mutations du motif. Par petites touches, l'ajout de détails réalistes va permettre de remettre progressivement en cause le modèle de la belle morte. Les derniers portraits ${ }^{43}$, symptôme de la nouvelle importance accordée à la mémoire des défunts, sont particulièrement touchés par ce cheminement, mais n'en sont pas moins astreintes à une exigence de ressemblance. Les marques les plus violentes de l'agonie et de la mort sont effectivement masquées, mais la particularité du genre du portrait rejette de facto l'idéalisation systématique des corps. Emmanuelle Héran ${ }^{44}$ a ainsi souligné le basculement engendré par le succès de l'œuvre de Léon Cogniet Le Tintoret peignant sa fille morte (1843) ${ }^{45}$. Si la toile ne présente pas une mimesis fidèle de la thanatomorphose, le corps n'est pas pour autant idéalisé. La bouche entrouverte et la carnation cireuse de la défunte apportent une note de trivialité qui ôte tout doute sur son état. 

d'insuffler des détails réalistes aux dépouilles féminines. Le spectaculaire est en effet un des leitmotivs des productions culturelles de cette période, en atteste le succès de la littérature macabre et des pièces les plus sombres sur le Boulevard du Crime ${ }^{46}$. Cette surenchère scénique et littéraire encourage l'émergence du "genre féroce ». Des œuvres aux sujets particulièrement sanglants essaiment sur les cimaises du Salon, participant au dévoiement des codes de la belle mort. Un bon exemple est offert par la toile Andromaque (1883) du peintre Antoine Rochegrosse ${ }^{47}$ (fig. 3), ovationnée au Salon de 1883. Pas moins de quinze corps particulièrement outragés (plusieurs têtes coupées et des pendus), dont celui d'une jeune femme, se partagent l'espace avec les huit protagonistes. Bien que cette dernière réponde aux canons de beauté de l'époque, sa pose affalée contre le mur et le sang séché derrière sa tête attestent la violence de son trépas. Sa nudité, accolée aux têtes coupées grimaçantes et aux larges flaques de sang, est d'ailleurs plus triviale qu'érotique.

Fig. 3.

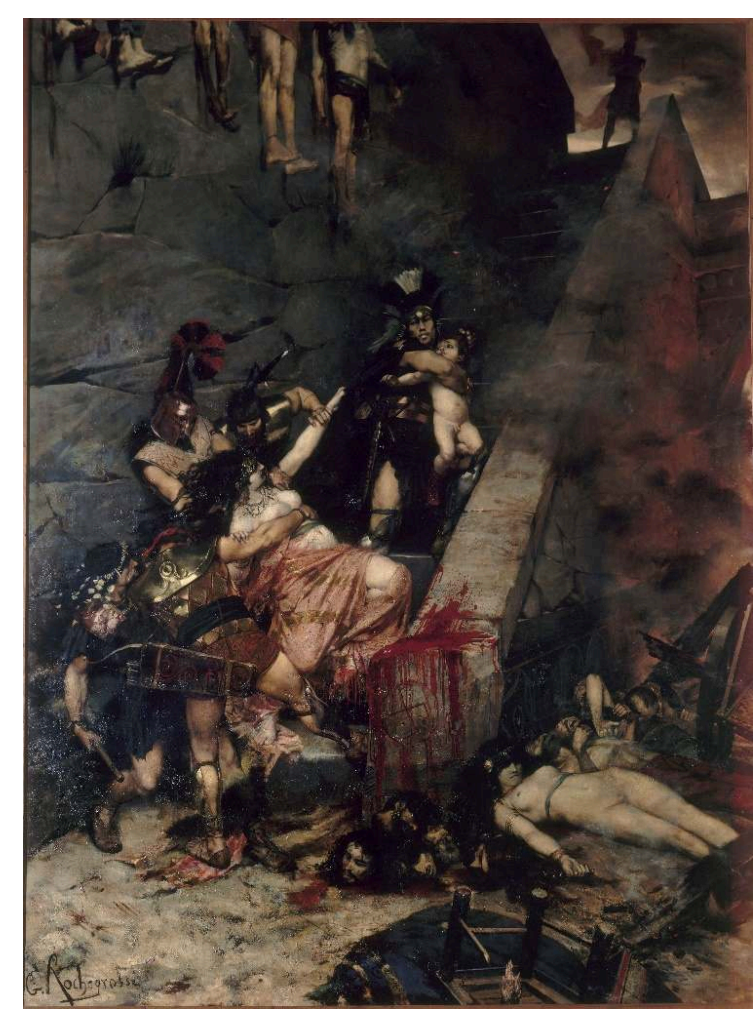

Georges-Antoine Rochegrosse, Andromaque, 1883, huile sur toile, Rouen, Musée des Beaux-Arts.

(c) RMN-Grand Palais / Agence Bulloz

21 Une dernière voie de dépassement des figures de belles mortes est discernable dans leurs pendants meurtriers. L'imaginaire des féminités mortifères est en effet très en vogue au $\mathrm{XIX}^{\mathrm{e}}$ siècle. Ces dernières sont incarnées par des figures historiques et mythologiques comme Salomé, Judith ou la Méduse, ou encore par des figures contemporaines liées à la sexualité, à l'instar des prostituées. L'aura négative associée à ces femmes permet aux artistes de se soustraire à l'injonction de peindre uniquement de belles défuntes. En témoigne la toile Persée, vainqueur de Méduse $(1867)^{48} \mathrm{du}$ peintre Eugène Thirion, laquelle présente un corps décapité bien loin de se prêter à une 
quelconque idéalisation. La Gorgone est ainsi figée dans une pose anguleuse, les poings encore serrés sous la violence de la bataille. Sa tête, dont le regard fixe le spectateur, offre quant à elle une carnation verdâtre et une plaie dégoulinante de sang.

À travers ces différents exemples se dessinent des relations bien plus complexes entre l'art et les stéréotypes de genre. La brèche que représente ces œuvres inaugure un basculement, encouragé par la remise en cause des normes de beauté et la recherche de réalisme qui traversent les arts au XIX ${ }^{e}$ siècle.

\section{Conclusion}

En 1914, Ferdinand Hodler réalise dix-huit tableaux et plus de cent-vingt dessins de l'agonie puis de la dépouille de sa maîtresse, Valentine Godé-Tarel. En refusant de magnifier son état et en confrontant le spectateur à la trivialité de la mort, cette série marque l'aboutissement d'une recherche esthétique de longue haleine. Rompant largement avec les normes de bienséance, l'artiste démontre l'intérêt de dépeindre un cadavre pour lui-même.

Les belles mortes sont pourtant loin de disparaître des cimaises du Salon. En témoigne le sujet du Prix de Rome de 1920, établi par l'Académie des Beaux-Arts d'après un vers du poète Lamartine : "Des pêcheurs un matin virent un corps de femme, Que la vague nocturne au bord avait roulé, Même à travers la mort sa beauté touchait l'âme ». Femme, mort et beauté sont de nouveau associées dans un triptyque qui a fait ses preuves.

25 La rapidité de cet exposé ne permet malheureusement pas de traiter la dimension temporelle de cette iconographie et de ses évolutions. Ce bref aperçu du motif de la belle morte dans la peinture du XIX ${ }^{e}$ siècle appelle cependant un constat. Si l'art est le reflet de son époque, produisant des œuvres qui embrassent les normes culturelles de son temps, il est également le terreau de leur rejet.

\section{NOTES}

1. Anne-Emmanuelle Demartini, Dominique Kalifa, Imaginaire et sensibilité au XIX ${ }^{e}$ siècle, études pour Alain Corbin, Paris, Creaphis, 2005, p. 5.

2. Le rapport au réel se joue sur deux échelles. La première est celle de la « vie » du cadavre (les changements qu'il subit lors des phases de cadavérisation puis de décomposition), et donc le choix de l'artiste de représenter le corps à un moment précis de cette évolution. La seconde est la fidélité ou non de la représentation de la thanatomorphose au moment de l'évolution choisi.

3. Ensemble des altérations morphologiques déterminées par la mort (définition du CNRTL).

4. Frédéric Cousinié, Beautés fuyantes et passagères, La représentation et ses «objets-limites » aux XVII XVIII ${ }^{e}$ siècles, Paris, Gérard Montfort, 2005, p. 200.

5. Charles Alphonse Du Fresnoy, De Arte Graphica, Paris, s. n., 1668, p. 69.

6. Thomas Gaehtgens, Christian Michel, Daniel Rabreau, Martin Schieder (dir.), L'art et les normes sociales au XVIII siècle, Paris, Maison des sciences de l'homme, 2001. 
7. Sur les 1500 œuvres que nous avons analysées, les femmes représentent environ un quart des dépouilles. Ce corpus se compose de peintures, dessins et estampes françaises comportant au moins un cadavre et s'inscrivant dans un période historique allant de 1808 à 1914 .

8. Paul Delaroche, La Jeune martyre, 1855, huile sur toile, $171 \times 148 \mathrm{~cm}$, Paris, Musée du Louvre.

9. Anne-Louis Girodet, Atala au tombeau, 1808, huile sur toile, 207 x $267 \mathrm{~cm}$, Paris, Musée du Louvre.

10. Collectif, Les Peintres de l'âme : art lyonnais du XIXe siècle, cat. exp., Lyon, Musée des Beaux-arts de juin à septembre 1981, Lyon, Édition du musée des Beaux-Arts de Lyon, 1981.

11. James Bertrand, Chrétiens retirant du Tibre les corps des martyrs, 1845, huile sur toile, $97 \times 147$ $\mathrm{cm}$, collection particulière.

12. Théodore Géricault, Têtes de suppliciés, 1818, huile sur toile, 50 x $61 \mathrm{~cm}$, Stockholm, National Museum.

13. Paul Cézanne, Le Meurtre, 1867, huile sur toile, $65 \times 80 \mathrm{~cm}$, Liverpool, National Museum.

14. Gotthold Ephraim Lessing, Laocoon, Paris, Hermann, 1990, p. 50.

15. Eugène Appert, Néron devant le cadavre d'Agrippine, 1824, huile sur toile, $247 \mathrm{x} 265 \mathrm{~cm}$, Montauban, Musée Ingres.

16. Antoine Jean Gros, Napoléon sur le champ de bataille d'Eylau, 1808, huile sur toile, $521 \times 784 \mathrm{~cm}$, Paris, Musée du Louvre.

17. Henri Regnault, Exécution sans jugement sous les rois Maures de Grenade, 1870, huile sur toile, 305 x $146 \mathrm{~cm}$, Paris, Musée d'Orsay.

18. Une seconde version de la «belle mort » masculine est issue des récits de mort glorieuse des héros grecs, au sein desquels le concept de Kalos Kagathos lie beauté des corps et des esprits. Cette dernière est largement reprise dans l'idéalisation néoclassique des corps masculins. Cependant ces morts faisant suite à la guerre, le rapport à la violence est très différent de celui des belles mortes. Voir Jean-Pierre Vernant, La Mort héroïque chez les Grecs, Nantes, Plein feux, 2001. J.-P. Vernant, La Mort dans les yeux : figures de l'autre en Grèce ancienne, Paris, Hachette, 1987.

19. Jean Pierre Granger, Apollon et Cyparisse, 1816, huile sur toile, $243 \times 189 \mathrm{~cm}$, Leipzig, Museum der Bildenden Künste.

20. Jules-Élie Delaunay, La Mort d'Abel, 1856, huile sur toile, 57 x $98 \mathrm{~cm}$, Paris, École nationale supérieure des beaux-arts.

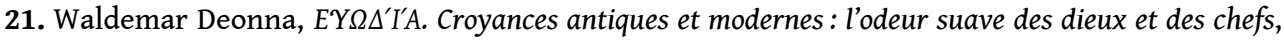
Turin, Nino Aragno, 2003.

22. Anne Carol, L'Embaumement, une passion romantique, Clamecy, Champ Vallon, 2015.

23. Philippe Ariès, Essai sur l'histoire de la mort en Occident du Moyen Âge à nos jours, Paris, Seuil, 1975. Michel Vovelle, La Mort et l'Occident de 1300 à nos jours, Paris, Gallimard, 1983.

24. A. Carol, Les Médecins et la mort XIXe-XXe siècle, Paris, Aubier, 2004. Alain Corbin, Le Temps, le désir et l'horreur, essai sur le XIXe siècle, Manchecourt, Champs Flammarion, 1998. A. Corbin, JeanJacques Courtine, Georges Vigarello (dir.), Histoire du corps, de la Révolution à la Grande Guerre, t. II, Paris, Seuil, 2005.

25. Réglementation des cimetières hors des villes, 1804. Réglementation du transport des cadavres de nuit et en voitures fermées, 1848 . Réglementation des abattoirs restreints à des lieux clos, 1806. Interdiction de l'épanchement public du sang animal, 1806. Réglementation des salles de dissection, 1803. Réglementation des exécutions tout au long du siècle.

26. A. Carol, op. cit. note 22.

27. Coralie De Gaix, CEuvres, Paris, Champion, 1912, p. 116.

28. Victor Hugo, Les Contemplations, Paris, Gallimard, 1990, p. 437-438.

29. Gustave Flaubert, Correspondance, 1830-1851, Paris, Gallimard, 1973, t. I, p. 258.

30. Léo Lespès, Les Yeux verts de la morgue, histoire à faire peur, Paris, Comptoir des imprimeurs unis, 1846, p. 205.

31. Théophile Gautier, La Morte amoureuse, Paris, Gallimard, 1992, p. 96. 
32. Amandine Malivin, Voluptés macabres, la nécrophile en France au XIX ${ }^{e}$ siècle, Thèse dirigée par Gabrielle Houbre, Université Paris 7, 2013.

33. Adolphe Lafferière, Souvenirs d'un jeune premier, Paris, Dentu, Paris, 1884, p. 43.

34. Carolyn Day, Consumptive chic: a history of beauty, fashion and disease, Londres, Bloomsbury, 2017.

35. Bram Dijkstra, Les Idôles de la perversité, Paris, Seuil, 1992.

36. Joëlle Bolloch, Emmanuelle Héran (dir.), Le Dernier portrait, cat. exp., Paris, Musée d'Orsay du 5 mars au 26 mai 2002, Paris, Réunion des Monuments Nationaux, 2002.

37. L. Poirel, Sarah Bernhardt dormant dans son cercueil, vers 1870, photographie sur papier albuminé, 10,7 x 6,5 cm, Paris, Bibliothèque Marguerite Durand.

38. Édouard Manet, Le Déjeuner sur l'herbe, 1863, huile sur toile, 207 x $265 \mathrm{~cm}$, Paris, Musée d'Orsay.

39. Henri Gervex, Avant l'opération, 1887, huile sur toile, 242 x $188 \mathrm{~cm}$, Paris, Musée d'Orsay.

40. Elisabeth Bronfen, Over Her Dead Body: Death, Femininity and the Aesthetic, Manchester, Manchester University Press, 1992.

41. Lisa Downing, Desiring the dead, necrophilia and Nineteen-century French literature, Oxford, Oxford University, 2003.

42. En 1772, le chirurgien Eugène Sue ouvre un « cours d'anatomie pittoresque sur le naturel » comprenant la dissection de cadavres. À sa mort en 1792, son fils Jean-Joseph Sue reprend son poste et fait construire un amphithéâtre pour pratiquer cette dernière au sein même du Louvre. La majorité des professeurs d'anatomie qui vont suivre au XIX ${ }^{\mathrm{e}}$ siècle vont également enseigner par le biais de la dissection. Voir Martial Guédron, «L'enseignement de l'anatomie artistique en France et la question de la dissection (XVIII ${ }^{\mathrm{e}}-\mathrm{XIX}{ }^{\mathrm{e}}$ siècles) », Les Cahiers d'histoire de l'art, $\mathrm{n}^{\circ} 2$ (2004), p. 33-40.

43. Portraits réalisés sur le lit de mort du défunt.

44. J. Bolloch, E. Héran (dir.), op. cit. note 36.

45. Léon Cogniet, Le Tintoret peignant sa fille morte, 1843, huile sur toile, $37,5 \mathrm{x} 46 \mathrm{~cm}$, Bordeaux, Musée des Beaux-Arts.

46. Juliette Goudot, «Pantomime et spectaculaire sur le Boulevard du Crime », pp. 245-252, dans Isabelle Moindrot (dir), Le Spectaculaire dans les arts de la scène du romantisme à la Belle Époque, Paris, CNRS édition, 2006, p. 78.

47. Georges-Antoine Rochegrosse, Andromaque, 1883, huile sur toile, 480 x 335 cm, Rouen, Musée des Beaux-Arts.

48. Eugène Romain Thirion, Persée, vainqueur de Méduse, 1867, huile sur toile, 240 x $135 \mathrm{~cm}$, Senlis, Musée d'Art et d'archéologie.

\section{RÉSUMÉS}

Allan Edgar Poe écrivait en 1856: "La mort d'une belle femme est incontestablement le plus poétique sujet du monde ». Si cette affirmation peut paraitre étrange de nos jours, elle témoigne de la longue prospérité du motif de la belle morte. Au XIX ${ }^{\mathrm{e}}$ siècle, ce dernier inonde les productions culturelles, au point de créer un imaginaire qui essaime aussi bien dans les journaux de mode que sur les cimaises du Salon. Ce succès est d'autant plus surprenant qu'il s'inscrit à contre-courant de l'évolution du reste des œuvres présentant des dépouilles. Or cette persistance 
est en partie issue de la représentation genrée des cadavres, qui rend le motif propice à l'expression des stéréotypes et de ce qu'ils impliquent concernant différents rapports à la violence. Les belles mortes sont donc un exemple de choix pour questionner la façon dont l'art est à la fois un espace de matérialisation et de dépassement des systèmes de pensée d'une époque.

In 1856 Allan Edgar Poe wrote: "The death of a beautiful woman is, unquestionably, the most poetical topic in the world". Although this statement may seem strange nowadays, it attests the high regard in which the motif of a beautiful dead woman was held. In the 19th century, this motif pervaded cultural works to the extent of creating an imaginative universe seen both in fashion reviews and on the walls of the Salon. This success is all the more surprising because it ran counter to the evolution of other works that featured dead bodies. This persistence derives in part from the gendered representation of corpses, which renders the motif suitable for expressing stereotypes and their implications regarding different relationships to violence. Beautiful dead women are therefore a perfect example for questioning how art is a space of both materialisation and the transcension of an era's systems of thought.

\section{INDEX}

Mots-clés : Cadavre, corps, violence, mort, femme, belle morte, belle endormie, genre, peinture, XIXe siècle, éros et thanatos, femme fatale

Keywords : Corpse, body, violence, death, woman, beautiful dead woman, sleeping beauty, gender, painting, 19th century, Eros and Thanatos, femme fatale

\section{AUTEUR}

\section{ANAELLE LAHAEYE}

Anaelle Lahaeye est diplômée de deux masters de l'Université Paris 1 Panthéon Sorbonne. Ses recherches, qui portent sur la représentation de la mort dans la peinture française du XIX ${ }^{\mathrm{e}}$ siècle, l'ont amenée à aborder les porosités du macabre entre théâtre et peinture romantique, puis à s'intéresser aux évolutions des normes de représentation des dépouilles. Elle mène actuellement une thèse sous la direction de Pierre Wat et est également chargée de cours en art contemporain et membre du groupe de recherche Studio XIX.

Anaelle Lahaye is diplomed from the Sorbonne University Paris 1 Panthéon (M.A.). Her research fields are : death representation in $19^{\text {th }}$ century french painting, the links between theater and romantic painting relating to death representation, the evolution of the iconographic codes in death representation. She works currently on her $\mathrm{PhD}$ thesis under the direction of Pierre Wat and teachs contemporary arts. She is also a member of the research group Studio XIX. 